\title{
The Case of a Job Well Done?
}

Asaf Acker ${ }^{1}$, Amir korngreen ${ }^{1}$, Bloom Shlomo', Shelef llan², Perry Zvi³, Atar Dan ${ }^{1}$ and David Lebel

${ }^{1}$ Orthopedic Department, Soroka University Medical Center, Rager Boulevard, P.O.B 151, Beer Sheva 84101, Israel

${ }^{2}$ Radiology Department, Soroka University Medical Center, Rager Boulevard, P.O.B 151, Beer Sheva 84101, Israel

${ }^{3}$ Department of Surgery "A", Soroka University Medical Center \& Epidemiology department, Faculty of Health Sciences Ben Gurion University of the Negev Beer Sheva 84101 , Israel

\begin{abstract}
Minimal invasive techniques for fracture repair are becoming more prevalent as increased experience is gained by orthopedic trauma surgeons worldwide, with minimal invasive plate osteosynthesis (MIPO) surgery being a popular one. In the current case report we describe a rare complication of fracture management, where anterior tibial artery occlusion occurred as a result of direct pressure by an LCP $4.5 \mathrm{~mm}$ plate inserted via MIPO technique.

The case involves a 41-year-old man was brought to the emergency trauma room after sustaining a gunshot injury to the Lt tibia. the surgical team preformed a closed reduction and internal fixation with LCP $4.5 \mathrm{~mm}$ (Synthes) plate and screws through a lateral incision in a minimal invasive technique. In the recovery room, thirty minutes after the end of the first surgery, the patient developed acute leg ischemia and was rushed back into the operating room. After lateral fasciotomy and exploration, the tibial peroneal tract was found to be completely torn, with no pulse of the tibialis anterior artery, Thrombectomy was performed and a graft used. Even though, the ischemia continued and Selective angiography demonstrated the tibialis anterior pressed between the tibia and the plate with complete obstruction. The plate was immediately removed and the artery was reconstructed using the saphenous vein from the uninjured leg. Even though arterial complications after fracture management are rare, they do happen and one should keep them in mind and be vigilant for them, thus if they do occur, one can attend to them early.
\end{abstract}

Keywords: Fracture; Minimal invasive plate osteosynthesis; Trauma; Tibia

\section{Introduction}

Minimal invasive techniques for fracture repair are becoming more prevalent as increased experience is gained by orthopedic trauma surgeons worldwide. Alongside with the increasing popularity of minimal invasive plate osteosynthesis (MIPO) surgery, the number of reported complications following minimal invasive procedures is increasing as well.

The documented complications of MIPO surgery include skin breakdown, infection, mal-union, delayed union, non-union and implant failure [1].

Vascular complications after treatment of tibial shaft fractures in general are quite rare, and comprise of direct arterial/venous damage, compartment syndrome and deep vein thrombosis (DVT) [2]. Most of the direct arterial injuries reported in the literature are sporadic case reports following external fixator or Ilizarov instrumentation placement [3,4], but also after intramedullary nailing of the Tibia [5] and even after arthroscopic treatment of ankle injury [6].

In the current case report we describe a rare complication of fracture management, where anterior tibial artery occlusion occurred as a result of direct pressure by an LCP $4.5 \mathrm{~mm}$ plate inserted via MIPO technique. As far as we know this is the first report of such a complication.

\section{Case Report}

A 41-year-old man was brought to the emergency trauma room after sustaining a high velocity gunshot injury to the Lt tibia. Prehospital care included tourniquet placement above the knee. On arrival, the patient was hemodynamically and respiratory stable, fully alert, Glasgow Coma Scale 15 and Revised Trauma Score of 12 (maximal scoring), with the only obvious sign of injury being the entry and exit wounds caused by the gunshot injury with an open fracture of the Left proximal tibia (Figure 1). General examination and initial trauma series radiographs were normal. Dorsalis pedis pulse was palpable and no ischemic damage was observed after removal of the tourniquet.

The patient was taken to the operating room, the wounds were debrided and irrigated. No tourniquet was used during surgery and there was no sign of ongoing heavy bleeding. Since severe proximal tibial damage was found, external fixator application was thought to be impossible without utilizing a cross knee technique. Therefore, the
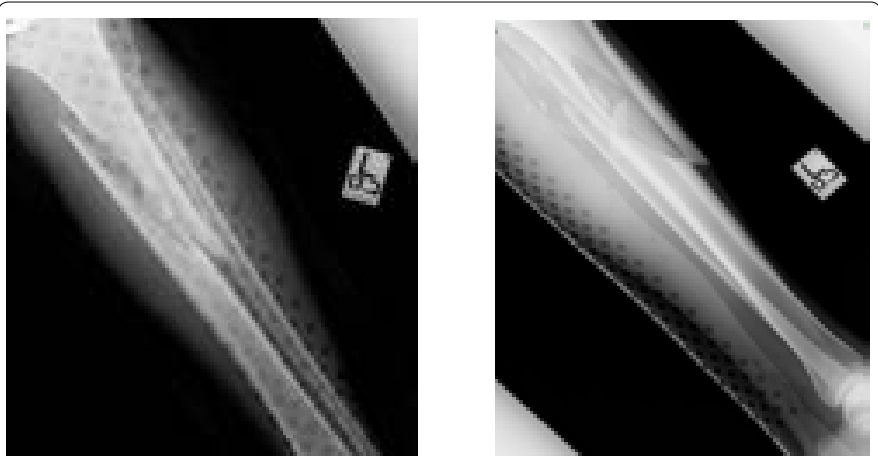

Figure 1: X-ray of Lt. tibia and fibula demonstrating an open fracture due to gun shoot injury, on arrival day.

*Corresponding author: Zvi Perry, Department of Surgery "A", Soroka University Medical Center, POB 151, Beer-Sheva, 84101 Israel, Tel: +972-8-640-0267; E-mail: zperry@bgu.ac.il

Received May 21, 2015; Accepted June 15, 2015; Published June 22, 2015

Citation: Acker A, korngreen A, Shlomo B, llan S, Zvi P, et al. (2015) The Case of a Job Well Done? J Trauma Treat 4: 257. doi:10.4172/2167-1222.1000257

Copyright: (C) 2015 Acker A, et al. This is an open-access article distributed under the terms of the Creative Commons Attribution License, which permits unrestricted use, distribution, and reproduction in any medium, provided the original author and source are credited. 
surgical team preformed a closed reduction and internal fixation with LCP $4.5 \mathrm{~mm}$ (Synthes) plate and screws through a lateral incision in a minimal invasive technique (Figure 2).

In the recovery room, thirty minutes after the end of the first surgery, the patient developed acute leg ischemia with immense pain, tachycardia and no distal pulses in the operated leg. The on-call vascular surgeon was called and the patient was rushed back into the operating room.

The second surgery was performed by orthopedic and vascular surgical teams. After lateral fasciotomy (done as a routine to relieve any possible pressure from the soft tissues) and exploration, the tibial peroneal tract was found to be completely torn, with no pulse of the tibialis anterior artery. Thrombectomy was performed with removal of some clots but there was no passage of the catheter within the tibialis anterior artery distally beyond $25 \mathrm{~cm}$. In order to minimize ischemic damage, prompt revascularization of the tibialis posterior artery with a sapheneous vein graft was preformed. At the end of the surgery a good pulse was palpable at the tibialis posterior point near the medial malleolus. To complete the surgery, the fasciotomy was enlarged to include all leg compartments, again, to exclude any possibility of vascular pressure by the soft tissues. The patient was transferred to the recovery room, again developed acute ischemic leg and was returned to the operating room.

Angiography was done on the operating table - it showed a small spastic tibialis posterior artery, and a decision was made to try and fix the anterior tibial artery as well. Selective angiography demonstrated the tibialis anterior pressed between the tibia and the plate with complete obstruction (Figure 3 ).
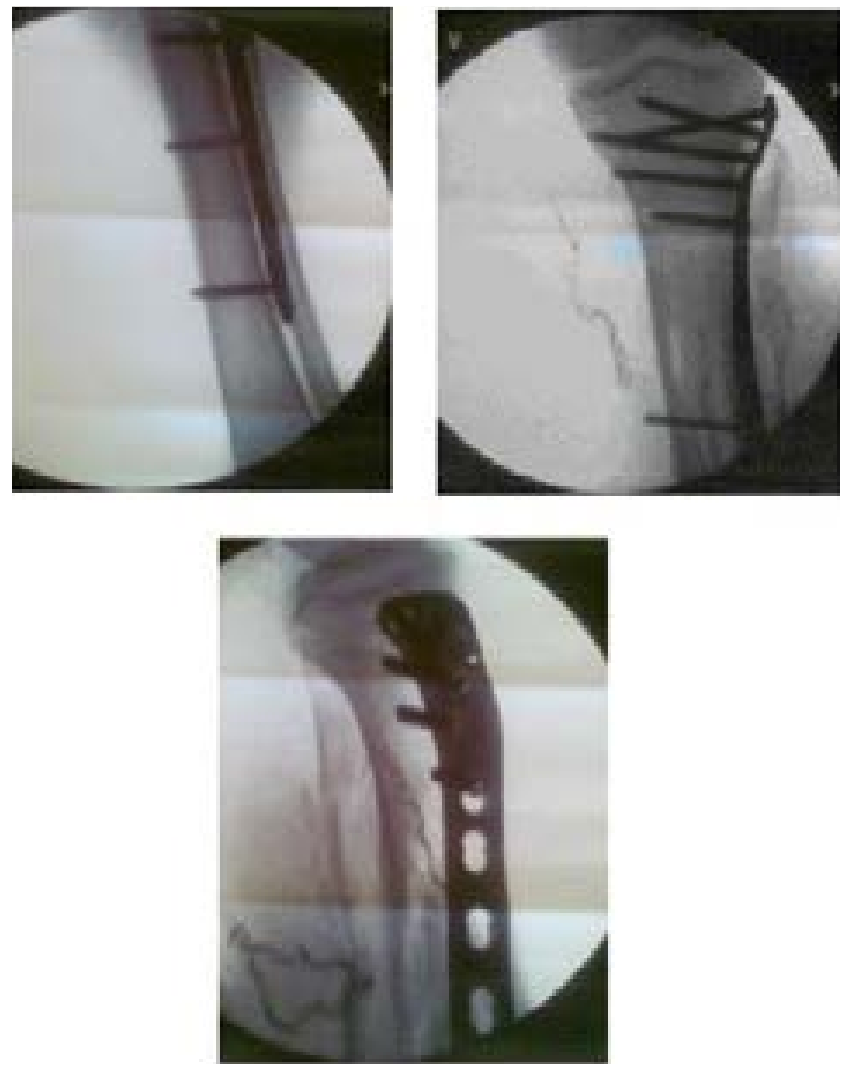

Figure 2: Post plating intraoperative X-ray.

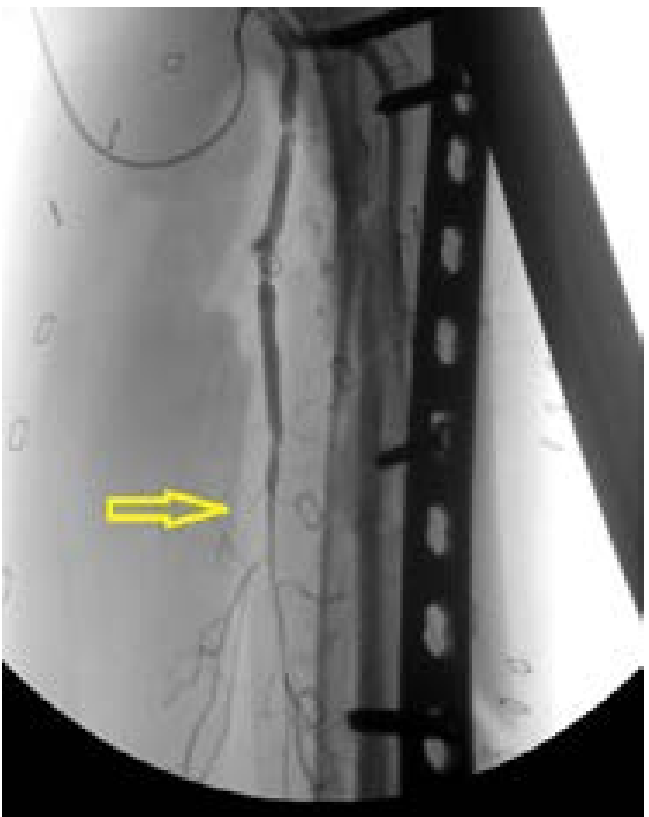

Figure 3A: Selective angiography demonstrating a spastic tibialis posterior artery (large arrow).

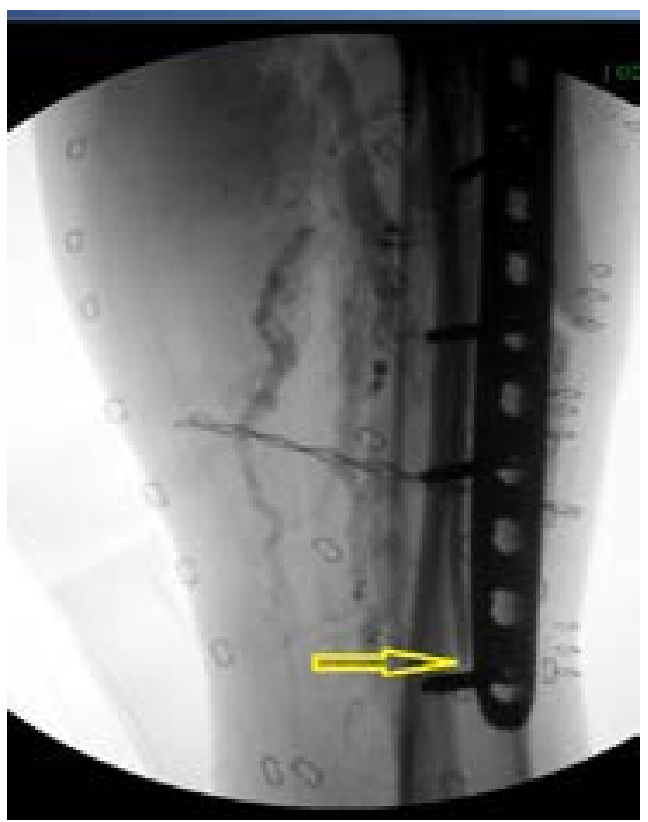

Figure 3B: Tibialis anterior artery compressed between the bone and the plate, showing a complete stop sign (arrow)

The plate was immediately removed and the artery was reconstructed using the saphenous vein from the uninjured leg (Figures 4 and 5). At the end of the surgery a good Doppler signal sounded on both arteries. A cross knee external fixator was placed to stabilize the fracture and the patient was taken to the intensive care unit.

Extubation was done the next morning and after another two days the patient was released from the ICU back to the Orthopaedic surgery department. No signs of ischemia were noticed during his hospitalization nor any neurologic deficits. 


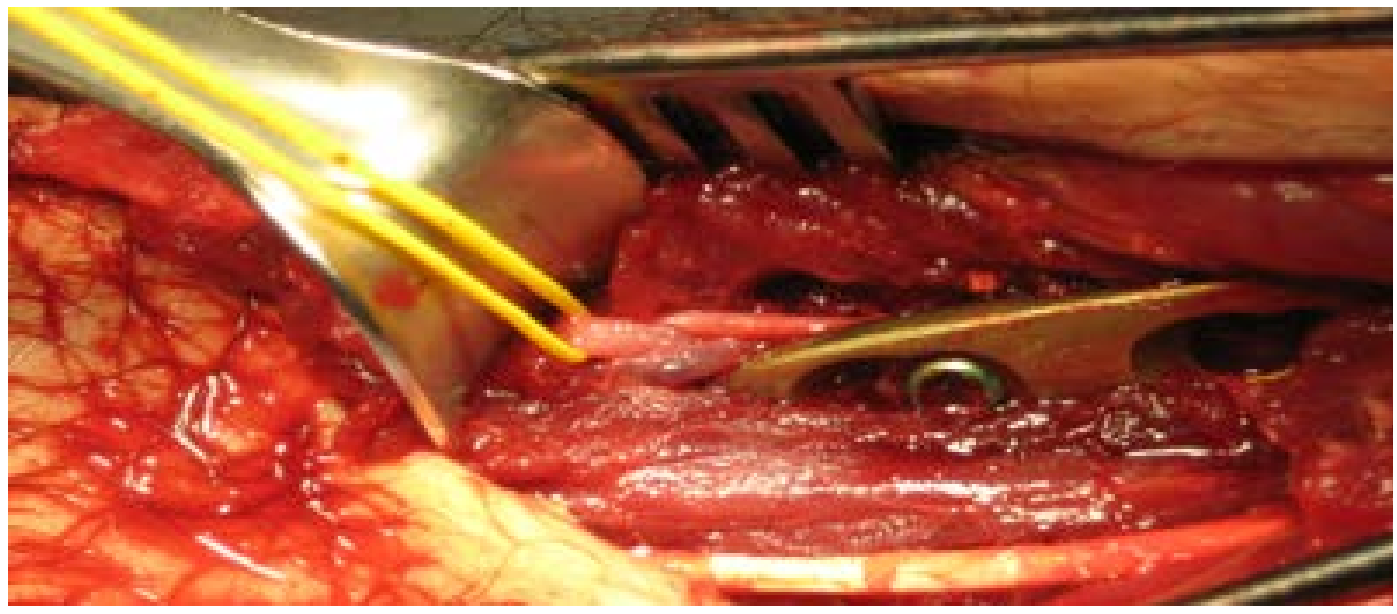

Figureg 4: Anterior tibial artery and vein compressed under the distal part of the plate.

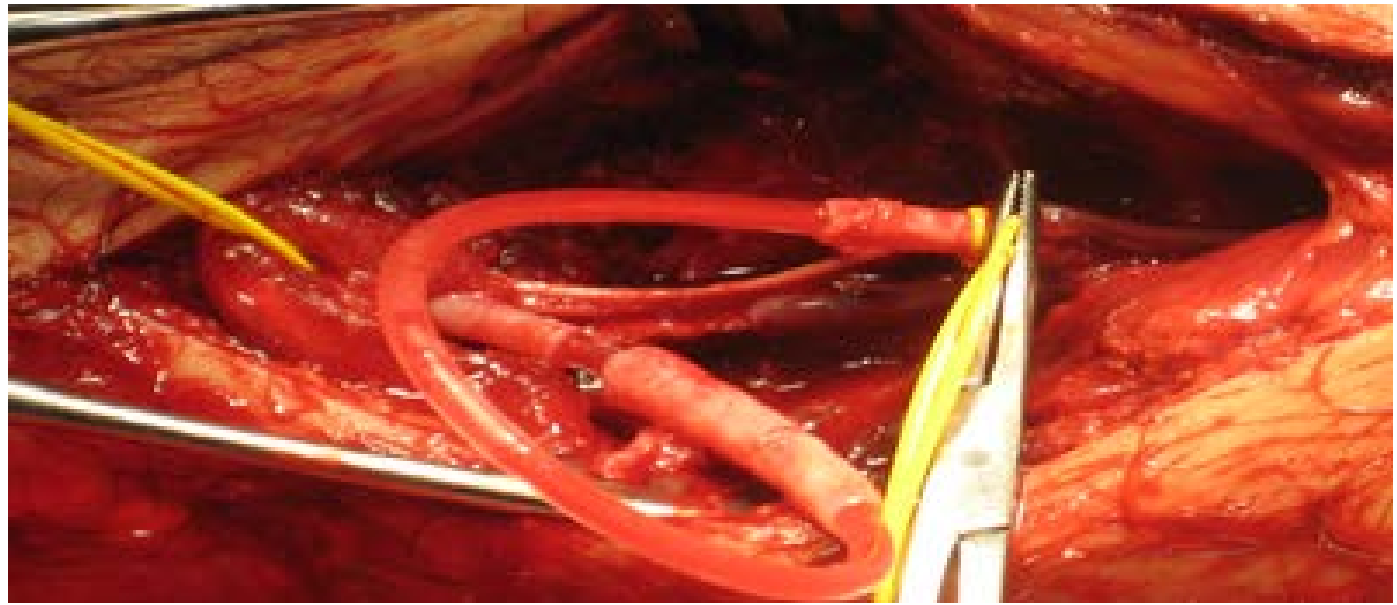

Figure 5: Reconstruction of the anterior tibial artery after plate removal.

After one week of in hospital treatment the patient self-discharged himself against medical advice $t$ before any definitive fracture managenet could be employed.. Since then, he was lost to follow up.

\section{Discussion}

The anatomic course of the anterior tibial artery (ATA) puts it in greater risk of injury during fracture surgery [7]. Proximally, the first arterial segment (from the bifurcation of the popliteal artery to the interosseous foramen) and the proximal part of the second segment (from the interosseous foramen to the level where the artery crosses the anterior border of the tibia) may be damaged when pin, wire or screw is directed posterolaterally at that level. Indamar et al [8] described a case report of a pseudoaneurysm formation in the proximal segment of the ATA after intramedullary nail and its interlocking screw insertion, which completely subsided after removal of the screw and nail. Case reports of impingement by external fixator pins have also been described [9].

In the distal part of the ATA, a straight mediolateral pin or wire may lacerate the artery or compress it. Papanikolaou et al. described the formation of a distal pseudoaneurysm after exchange of external fixator pins [4], while Ladermann et al. [10] reported the formation of a pseudoaneurysm 7 months after open reduction and external fixation of distal tibia fracture by plate and screws.

Van Hensbroek et al. [11] described the same phenomena after only 6 weeks of their MIPO surgery with an LCP plate. They also described an endovascular intervention and repair of the aneurysm.

In our case, no pseudoaneurysm developed, rather true ischemia appeared shortly after plate fixation, as a result of direct plate compression over the ATA, and as in the latter case report by Van Hensbroek et al. [11] vascular surgery intervention was mandatory.

The MIPO technique is based on a small incision followed by supra-periosteal canal preparation, and finally plate insertion and fixation by screws via small stab incisions, all done under fluoroscopic imaging guidance. Most common complications of MIPO surgery are not intraoperative in nature but rather post-operative: union problems, infections and implant failure [12]. We have described a unique intraoperative complication of MIPO surgery that was not described before in the literature. A possible explanation for the development of that specific complication in our case report might reside in the fact that both tibialis posterior artery and tibialis anterior artery were damaged one from the primary injury and the other from the plate. It is possible 
that injury to the ATA happens more often than we acknowledge but the damage is not clinically obvious because of patency of the tibialis posterior artery.

Even though this complication in extremely rare, we think that it is most likely preventable. The direct pressure of the plate on the ATA could have been noticed sooner during the first surgery if a larger distal incision would have been used, instead of a stab incision, and a visual confirmation of the distal contact area between the bone and the plate was carefully examined with no soft tissue entrapment verified. Another useful option would have been to use a Doppler device to verify the existence of distal pulses at the end of the plating.

Since we didn't find in the literature any similar report regarding vascular complication of the MIPO technique, we found it important to report of such a complication although follow up was not complete.

\section{Conclusion}

Vascular injury is a rare but a possible complication of MIPO surgery, and the orthopedic surgeon must be aware of such a complication and its implications.

\section{References}

1. Reudi TP, Murphy WM (2000) AO Principals Of Fracture Management, Thieme, Italy.

2. Browner DB, Levine AM, Jupiter JB, Trafton PG, Krettek C (2008) Skeletal Trauma, 4th edition, Vol. 2. Elsevier, Philadelphia.
3. Lim EV, Lavadia WT, Blebea J (1995) Vascular impingement by external fixator pins: A case report. J Trauma 38: 833-835.

4. Papanikolaou A, Thanassas C, Arealis G, Maris J (2008) External fixation and pseudoaneurysm: report of a case related to tibial lengthening. Orthopedics 31 .

5. Craxford S, Karuppiah SV, Milner S (2013) Pseudoaneurysm of the anterior tibial artery following tibio-talar-calcaneum fusion with a retrograde nail: A rare case and literature review. Case Reports in Orthopedics.

6. Jacobs E, Groot D, Das M, Hermus JP (2011) Pseudoaneurysm of the anterior tibial artery after ankle arthroscopy. J Foot Ankle Surg 50: 361-363.

7. Ebraheim NA, Lu J, Hao Y, Biyani A, Yeasting RA (1998) Anterior tibial artery and its actual projection on the lateral aspect of the tibia: a cadaveric study. Surg Radiol Anat 20: 259-262.

8. Inamdar D, Alagappan M, Shyam L, Devadoss S, Devadoss A (2005) Pseudoaneurysm of anterior tibial artery following tibial nailing: a case report. $J$ Orthop Surg (Hong Kong) 13: 186-189.

9. Mercer NS, Moss AL (1989) Impingement of vascular pedicles by externa fixator pins. Injury 20: 114-116.

10. Lädermann A, Stern R, Bettschart V, Riand N (2008) Delayed posttraumatic pseudoaneurysm of the anterior tibial artery mimicking a malignant tumor. Orthopedics 31: 500 .

11. van Hensbroek PB, Ponsen KJ, Reekers JA, Goslings JC (2007) Endovascular treatment of anterior tibial artery pseudoaneurysm following locking compression plating of the tibia. J Orthopedic Trauma 21: 279-282.

12. Kehr P, Graftiaux A (2006) Master techniques in orthopaedic surgery: Fractures, 2nd edn, Wiss DA (ed) Lippincott Williams \& Wilkins, USA 